\title{
SOX9 overexpression plays a potential role in idiopathic congenital talipes equinovarus
}

\author{
ZHENGDONG WANG $^{1,2}$, NAN YAN $^{2}$, LIYING LIU $^{1,3}$, DONGHUA CAO $^{1}$, \\ MING GAO ${ }^{1}$, CHANGKUN LIN ${ }^{1}$ and CHUNLIAN JIN ${ }^{1}$ \\ ${ }^{1}$ Department of Medical Genetics, China Medical University, Shenyang 110001; ${ }^{2}$ Department of Anatomy, \\ Shenyang Medical College, Shenyang 110034; ${ }^{3}$ Shenyang Women's and Children's Hospital, Shenyang 110001, P.R. China
}

Received July 29, 2012; Accepted December 4, 2012

DOI: $10.3892 / \mathrm{mmr} .2012 .1245$

\begin{abstract}
The collagen, type IX, alpha 1 (COL9AI) gene was previously identified as a candidate gene for idiopathic congenital talipes equinovarus (ICTEV), a congenital lower limb deformity in humans. In the present study, increased expression levels of COL9Al were identified in the abductor hallucis muscle of ICTEV patients compared with those in control samples. The COL9A1 gene is regulated by SRY (sex-determining region $Y$ )-box 9 (SOX9). Immunofluorescence analysis of SOX9 and COL9A1 proteins identified colocalization to the sarcolemma, endomysium and muscle membrane in muscle samples of ICTEV. No mutations in the exons and promoters of SOX 9 were detected in blood samples of 84 ICTEV patients by denaturing gradient gel electrophoresis. mRNA and protein expression levels of SOX 9 were detected by real-time polymerase chain reaction and western blot analysis, respectively and were found to be significantly higher in ICTEV muscle samples compared with those in control samples. Based on present observations, we hypothesize that overexpression of the SOX9 gene may play a role in the genetic etiology of ICTEV.
\end{abstract}

\section{Introduction}

Every year, $0.3-0.7 \%$ of live births worldwide are affected by congenital limb deformities caused by abnormalities in soft tissue which lead to varus (inclined inward), adductus (axially rotated) and equinus (pointed downward) fixation of the foot. This limb deformity is known as idiopathic congenital talipes equinovarus (ICTEV) (1-2). Neurological, muscular, bony, vascular and connective tissue mechanisms have been hypothesized to be involved in the pathology of ICTEV; however, the specific roles of these mechanisms in ICTEV

Correspondence to: Professor Chunlian Jin, Department of Medical Genetics, China Medical University, Bei'er Road Shenyang 110001, P.R. China

E-mail: chunlianjin@126.com

Key words: clubfoot, congenital, gene expression, SRY (sex-determining region Y)-box 9 remain unclear. Previous studies in twins and various ethnicities, as well as pedigree analyses, have indicated that a genetic basis in combination with additional environmental factors is responsible for ICTEV (2-6). It is becoming increasingly clear that genetic factors are important for the pathogenesis of ICTEV. Furthermore, genes associated with limb development, including homeobox D13 (HOXD13), collagen, type IX, alpha 1 (COL9A1) and GLI family zinc finger 3 (GLI3), are significantly associated with ICTEV (7-9).

We previously performed transmission disequilibrium test analysis, identifying that the COL9A1 gene on chromosome $6 \mathrm{q} 12-13$ is associated with ICTEV. Increased expression of COL9A1 was identified in abductor hallucis muscle samples of ICTEV patients compared with control samples, indicating that the COL9A1 gene may be an important susceptibility gene for ICTEV (7). Mutation screening experiments found no mutations in the exons and the upstream sequence of the COL9A1 gene in ICTEV patients. ICTEV patients may exhibit increased expression of $C O L 9 A 1$ but not with mutations in the exons and the upstream regions of $C O L 9 A 1$ (7). At present, the cause of the high expression levels of COL9A1 in muscle samples of ICTEV patients remains unclear.

Specific in vitro and in vivo studies have revealed that the SRY (sex-determining region Y)-box 9 (SOX9) transcription factor binds to consensus sequence pairs in the upstream region of the COL2A1 and COL9AI genes and regulates expression (10-16). SOX9 is a protein of the HMG-box DNA-binding protein class, which recognize the CCTTGAG sequence. SOX 9 is important for chondrocyte differentiation and the transcriptional regulation of the Anti-Müllerian Hormone, as well as steroidogenic factor-1 (17). Deficiencies in $S O X 9$ result in a skeletal malformation syndrome (camptomelic dysplasia) and are also frequently associated with sex reversal (18). In a previous study, expression of COL9A1 was found to be regulated by $S O X 9$ in chondrocytes (14). Thus, we hypothesized that there is a correlation between $S O X 9$ and high expression levels of COL9A1 in muscle samples of ICTEV patients.

\section{Materials and methods}

Patients and normal individuals. Abductor hallucis muscle samples were obtained from 15 ICTEV patients (12 males and 3 females) aged 4-12 years (average, 6.6 years old). Medial 
Table I. PCR primer sequences of SOX9.

\begin{tabular}{|c|c|c|c|c|}
\hline Region & Direction & Primer sequence $\left(5^{\prime}->3^{\prime}\right)$ & $\begin{array}{c}\text { Annealing } \\
\text { temperature }\left({ }^{\circ} \mathrm{C}\right)\end{array}$ & Product size (bp) \\
\hline Promoter 1 & $\begin{array}{l}\mathrm{F}^{*} \\
\mathrm{R}\end{array}$ & $\begin{array}{l}\text { TTACAAACCAAGTGACCGGC } \\
\text { TGCCTGCAAAAGTGCTTAGA }\end{array}$ & 55 & 495 \\
\hline Promoter 2 & $\begin{array}{l}\mathrm{F}^{*} \\
\mathrm{R}\end{array}$ & $\begin{array}{l}\text { TTATTACGGAGGAACAGCGG } \\
\text { CTTTCGGCTCTCCAACTCC }\end{array}$ & 62 & 588 \\
\hline Promoter 3 & $\begin{array}{l}\mathrm{F}^{*} \\
\mathrm{R}\end{array}$ & $\begin{array}{l}\text { GCTCTAAGGTGAGGCGGAGT } \\
\text { ATGAAGGGGTCCAGGAGATT }\end{array}$ & 56 & 445 \\
\hline Exon 1 & $\begin{array}{l}\mathrm{F}^{*} \\
\mathrm{R}\end{array}$ & $\begin{array}{l}\text { GCTGGTTTGAGAGGCAGAAA } \\
\text { CAACACAGAGAATATGACCCCA }\end{array}$ & 58 & 497 \\
\hline Exon 2 & $\begin{array}{l}\mathrm{F}^{*} \\
\mathrm{R}\end{array}$ & $\begin{array}{l}\text { CTTCAGCCATGGACAGTTCC } \\
\text { CAACTCCCTTCTCTGGCTGT }\end{array}$ & 58 & 340 \\
\hline Exon 3 & $\begin{array}{l}\mathrm{F}^{*} \\
\mathrm{R}\end{array}$ & $\begin{array}{l}\text { ACTCCGCCAGAGTGGAGCGT } \\
\text { TTTACGCGCCTGGAGCGAGC }\end{array}$ & 58 & 573 \\
\hline
\end{tabular}

"GC clamp was added at the 5' end of the forward primers. GC clamp: cgccegecgegeccegegeceggecegecgeccecgeccg. F, forward; R, reverse; SOX9, SRY (sex-determining region Y)-box 9.

plantar release was performed on the patients following failure of conservative treatment. Abductor hallucis muscle control samples were obtained from 2 fresh cadavers (2 males) and 7 trauma patients ( 3 males and 4 females) aged 5-11 years (average, 7.5 years old). All fresh samples were stored at $-80^{\circ} \mathrm{C}$ as soon as possible. Peripheral blood samples were obtained from 84 ICTEV patients (50 males and 34 females) aged 3-12 years (average, 6.2 years old). The blood samples were stored at $-20^{\circ} \mathrm{C}$. All patients were recruited at the Department of Pediatric Orthopedic Surgery (Second Affiliated Hospital of China Medical University, Shenyang, China). Informed consent was obtained from the parents of the patients and the study was approved by the China Medical University Ethics Committee. The probands revealed the typical ICTEV phenotype [varus (inclined inward), adductus (axially rotated) and equinus (pointed downward) fixation of the foot].

Analysis of SOX9 and COL9A1 expression in muscle samples by immunofluorescence using confocal laser scanning microscopy. Muscle samples were embedded in paraffin and fixed in $4 \%$ paraformaldehyde overnight at $4^{\circ} \mathrm{C}$. Tissues were sectioned into $4-\mu \mathrm{m}$ slices. Specific slides were stained with hematoxylin and eosin (H\&E). Immunofluorescence was performed according to the manufacturer's instructions (Wuhan Boster Biological Technology, Ltd., Wuhan, China). To quench nonspecific binding, sections were incubated in normal goat serum for $1 \mathrm{~h}$ at room temperature. Sections were then incubated in rabbit anti-human SOX9 polyclonal and mouse anti-human COL9A1 monoclonal antibodies (both 1:50; Santa Cruz Biotechnoloy, Inc., Santa Cruz, CA, USA) overnight at room temperature and washed 3-4 times in 0.01 mol/1 PBS. Sections were hybridized with 4',6-diamidino-2-phenylindole, FITC-labeled goat anti-rabbit immunoglobulins and trimethyl rhodamine isothiocyanate-labeled goat anti-mouse immunoglobulins (all 1:1,000; Wuhan Boster Biological Technology, Ltd., Wuhan, China) for $1 \mathrm{~h}$ at $37^{\circ} \mathrm{C}$ in the dark and then washed 3-4 times in $0.01 \mathrm{~mol} / \mathrm{l} \mathrm{PBS}$. Confocal laser scanning microscopy (CLSM) was used to analyze protein expression of SOX9 and COL9A1.

Mutational analysis of the SOX9 gene in blood samples by denaturing gradient gel electrophoresis (DGGE). Genomic DNA was extracted from 84 fresh blood samples obtained from ICTEV patients using a blood DNA extraction kit (Tiangen Biotech, Beijing, China) according to the manufacturer's instructions. Six primer pairs (presented in Table I) were designed to amplify SOX9 exons 1-3 and the 5' flanking sequence. Polymerase chain reaction (PCR) products of SOX9 were detected by DGGE, a sensitive method to separate alleles based on differences in melting behavior (19). DNA was visualized following ethidium bromide staining by UV transillumination and images were captured using a Polaroid camera.

Analysis of SOX9 mRNA expression in muscle samples by real-time PCR (RT-PCR). RT-PCR was performed to evaluate differences in RNA expression levels of SOX9. RNA was extracted from the muscle samples using a tissue RNA kit (Tiangen, China) and cDNA was synthesized using a reverse transcription kit (Promega Corporation, Madison, WI, USA) according to the manufacturer's instructions. RT-PCR amplification was performed in $25-\mu 1$ reaction volumes with $12.5 \mu \mathrm{l}$ SYBR Premix Ex Taq, $9.5 \mu \mathrm{l}$ deionized water, $0.5 \mu \mathrm{l}$ (initial concentration, $10 \mu \mathrm{M}$ ) of each primer and $2 \mu \mathrm{l}$ cDNA according to the manufacturer's instructions (Advanced Biotechnologies Inc., Columbia, MD, USA). Amplification was performed by pre-denaturation at $95^{\circ} \mathrm{C}$ for $10 \mathrm{sec}$, followed by 40 rounds of denaturation at $95^{\circ} \mathrm{C}$ for $5 \mathrm{sec}$ and annealing and extension at $58^{\circ} \mathrm{C}$ for $20 \mathrm{sec}$. Primer sequences were as follows: SOX9, 5'-ACT CGC CAC ACT CCT CCT C -3' (forward) and 5'-CCC TCT CGC TTC AGG TCA-3' (reverse); $\beta$-actin, 5'-CCC AGA GCA AGA GAG GCA-3' (forward) and 5'-GGG 
A
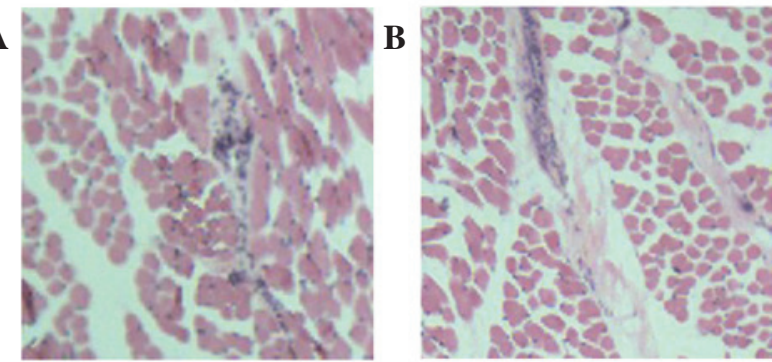

Figure 1. H\&E staining of muscle samples. Muscle samples of (A) ICTEV and (B) control. H\&E staining demonstrated the difference between the two groups (magnification, x100). Disorder in the tissue structure of the patients muscle samples is presented. Fibrosis of the muscle tissue is hypothesized to be due to the proliferation of mesenchymal cells. ICTEV, idiopathic congenital talipes equinovarus. $\mathrm{H} \& \mathrm{E}$, hematoxylin and eosin.
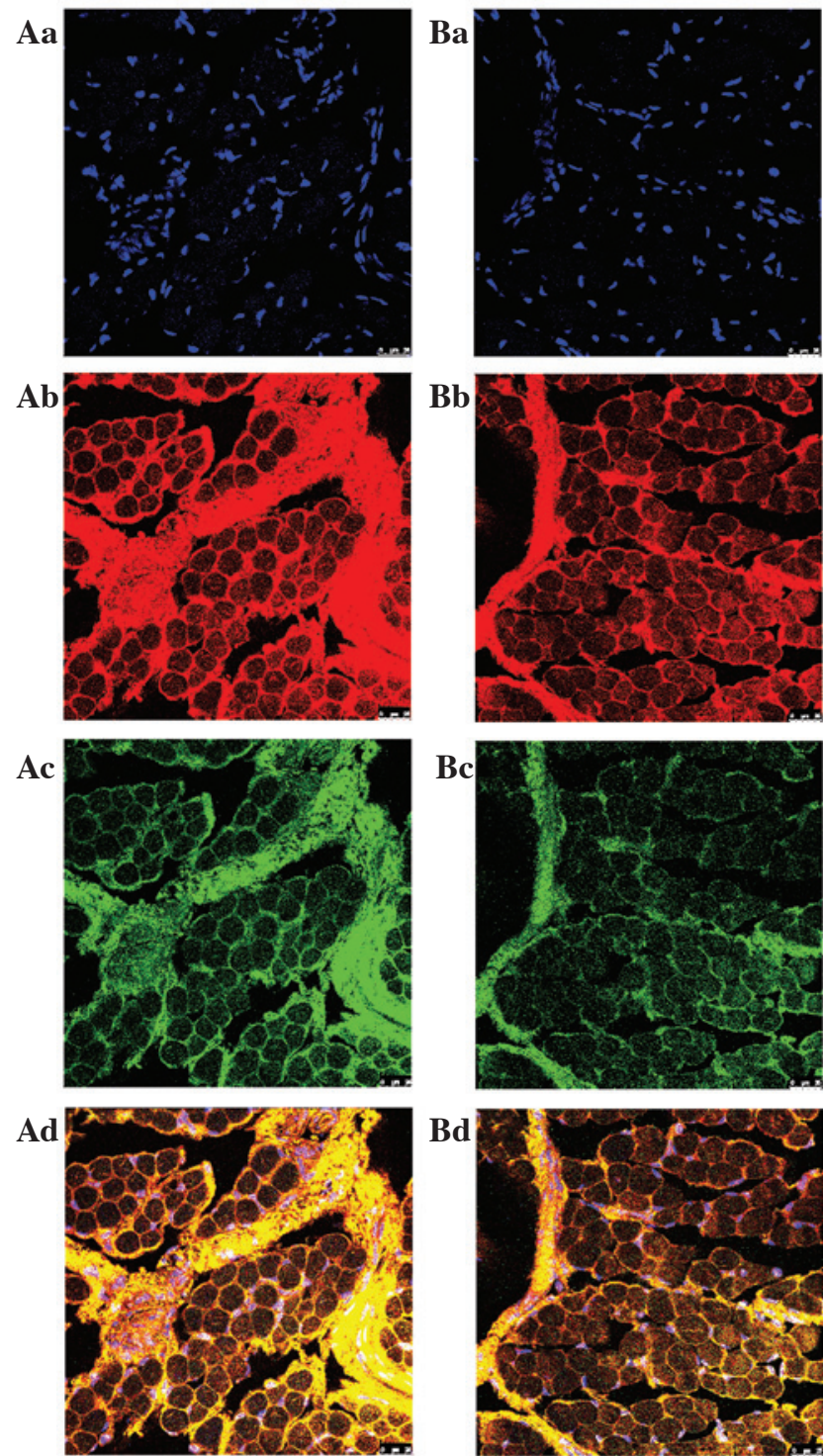

Figure 2. Analysis of SOX9 and COL9A1 in abductor hallucis muscle samples of ICTEV patients by immunofluorescence using CLSM. (A) ICTEV patients and (B) control. (a) Blue fluorescence, nuclei; (b) red fluorescence, COL9A1 protein; (c) green fluorescence, SOX 9 protein; and (d) yellow fluorescence, merged images. High expression of SOX9 and COL9A1 was colocalized to the connective tissue of the sarcolemma, endomysium and muscle membrane of the ICTEV patient (magnification, x400). ICTEV, idiopathic congenita talipes equinovarus; COL9A1, collagen, type IX, alpha 1; SOX9, SRY (sex-determining region Y)-box 9

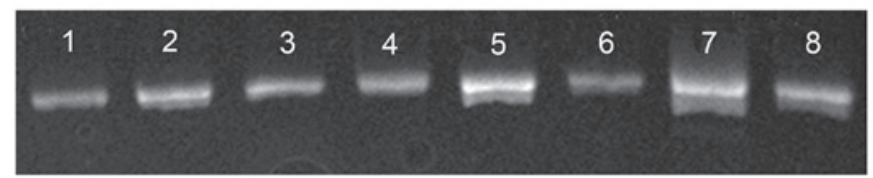

Figure 3. Analysis of PCR products by DGGE. A representative result of analysis of exon 1 of SOX9 is presented. Lanes 1-7, blood samples from ICTEV patients; and 8, normal control blood sample. DGGE gels (20-50\%) revealed no mutations in the samples. ICTEV, idiopathic congenital talipes equinovarus; DGGE, denaturing gradient gel electrophoresis.

AGC CAC ACG CAG-3' (reverse). Data were analyzed using the $2^{-\Delta \Delta C T}$ method.

Analysis of SOX9 protein expression in muscle samples by western blot analysis. Western blot analysis was used to evaluate levels of $S O X 9$ protein expression. Cytoplasmic protein was extracted from the abductor hallucis muscle samples from ICTEV patients and controls using a cytoplasmic and nuclear protein extraction kit (Active Motif, Carlsbad, CA, USA) according to the manufacturer's instructions. The protein concentration was determined spectrophotometrically (Unico, Dayton, NJ, USA) at $280 \mathrm{~nm}$ (20). Sample buffer (Beyotime Institute of Biotechnology, Jiangsu, China) was added to the cytoplasmic protein samples and the solution was loaded onto a $6 \%$ polyacrylamide gel. Following protein separation, the polyacrylamide gel was electroblotted onto a PVDF membrane (Millipore, Billerica, MA, USA). Nonspecific binding sites were blocked with $3 \%$ bovine serum albumin (Sigma, Poole, UK) in TBST buffer [20 mM Tris-buffered saline, $0.047 \%$ Tween ( $\mathrm{pH} 7.4)]$ overnight at $4^{\circ} \mathrm{C}$. The membrane was incubated for $3 \mathrm{~h}$ with rabbit anti-human SOX9 polyclonal antibody (1:50; Santa Cruz Biotechnoloy, Inc.) at room temperature and washed for $40 \mathrm{~min}$ in TBST. The membrane was then incubated for $2 \mathrm{~h}$ at room temperature with a goat anti-rabbit IgG horseradish peroxidase-conjugated antibody (1:4,000; Davis, CA, USA). Protein bands were visualized using modified enhanced chemiluminescence (Tiangen Biotech, China). Quantification of relative band densities was performed using standard densitometry scanning techniques.

Statistical analysis. Data are expressed as mean \pm SD and analyzed with SPSS (v13.0, SPSS, Inc., Chicago, IL, USA). Differences between two groups were analyzed using the Student's t-test. $\mathrm{P}<0.05$ was considered to indicate a statistically significant difference. Correlation was estimated using the Spearman's rank method.

\section{Results}

Light microscopy revealed disorder in the tissue structure of the H\&E-stained muscle samples. The presence of increased fibrous tissue in the ICTEV muscle samples was indicative of fibrosis (Fig. 1). Immunofluorescence by CLSM revealed that high expression of SOX9 and COL9A1 was colocalized to the connective tissue of the sarcolemma, endomysium and muscle membrane of ICTEV patients (Fig. 2). The results indicated that increased expression of COL9A1 may be associated with SOX9. No mutations were observed in the 5 ' flanking sequence 


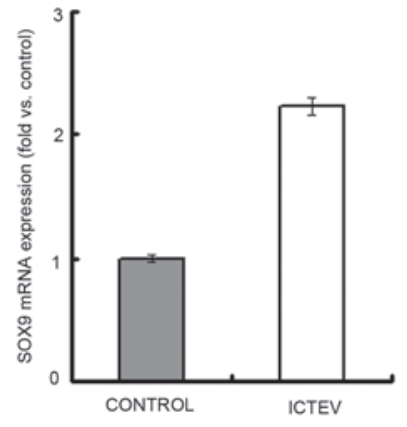

Figure 4. mRNA expression levels of $S O X 9$ in abductor hallucis muscle samples of ICTEV and control cases were analyzed by RT-PCR. Data were analyzed using the $2^{-\triangle \Delta C T}$ method. Results represent the fold increase vs. control. RNA expression levels of SOX9 in the ICTEV group were significantly higher than those in the control samples. $\mathrm{P}<0.05$, vs. control medium, by Student's t-test. ICTEV, idiopathic congenital talipes equinovarus; SOX9, SRY (sex-determining region Y)-box 9; RT-PCR, real-time polymerase chain reaction.

A

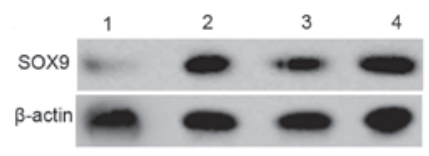

B

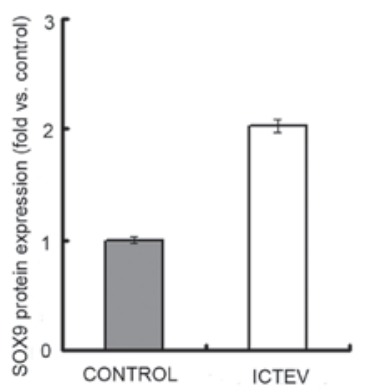

Figure 5. Analysis of SOX 9 protein expression in cytoplasm extracts from the abductor hallucis muscle samples of ICTEV and control patients by western blot analysis. (A) Two representative western blots are presented. Lanes 1 and 3, control; and 2 and 4, ICTEV patients. (B) Densitometric quantification of SOX9 in the muscle samples. Fold increase vs. control is indicated. Compared with control, $S O X 9$ expression was significantly higher in muscle samples of ICTEV. P<0.05, vs. the corresponding control. ICTEV, idiopathic congenital talipes equinovarus; SOX9, SRY (sex-determining region Y)-box 9

or exons 1-3 of SOX9 in the blood samples of 84 ICTEV patients (Fig. 3). As demonstrated in Figs. 4 and 5, RNA and protein expression levels of $S O X 9$ in the case samples were $\sim 2$ fold those of the controls. The correlation between mRNA and protein expression was positive, with a Spearman's rank correlation coefficient of 0.58 .

\section{Discussion}

Varus foot deformity in ICTEV patients is believed to be caused by contracture of medial soft tissue in the foot, including the deltoid and talonavicular ligaments and posterior tibial muscle. An additional cause of varus foot may be the shortening of the soft tissue at the bottom of the foot, including the plantar fascia, abductor hallucis and flexor digitorum brevis. Medial plantar release is a recognized surgical technique for the treatment of clubfoot. However, the technique is reserved for children who have already reached walking age and have no reducible deformity or those for whom conservative treatment has failed. The abductor hallucis muscle samples are the contracture tissues removed from the ICTEV patients by medial plantar release. Fibrosis was observed under light microscope in the connective tissues of the muscle samples from ICTEV patients. In a previous study, Ippolito and Ponseti hypothesized that retraction fibrosis of the distal muscles occurs in ICTEV patients (21). In addition, Ippolito identified increased fibrosis of muscle tissue in 4 aborted fetuses with clubfoot (22). It is generally accepted that fibrosis of muscle tissue is often caused by increased collagen components. Ionasescu et al also identified increased collagen synthesis in clubfeet (23).

Our previous study revealed that genes on chromosome 6q12-13 may be associated with ICTEV. Further analysis also indicated that $C O L 9 A 1$, in this chromosomal region, is a candidate gene for ICTEV based on extended transmission disequilibrium testing and was expressed in significantly higher quantities in the abductor hallucis muscle of ICTEV compared with control samples. These observations indicated that the COL9A1 gene may be an important susceptibility gene in the development of ICTEV. The COL9AI gene encodes one of the three $\alpha$ chains of type IX collagen, which is a minor collagen component that makes up 5-20\% of cartilage as well as type II collagen. Type IX collagen gene mutations result in multiple epiphyseal dysplasia with osteochondritis dissecans and a mild myopathy (24). A previous study in which intramuscular injection of bone morphogenetic proteins was performed to induce ectopic bone formation demonstrated that non-myogenic cells residing in the fascia of skeletal muscle have a marked chondrogenic potential and may represent a novel donor cell source for cartilage regeneration and repair (25).

In the present study, increased levels of COL9A1 protein were identified in the connective tissue of the epimysium, sarcolemma, endomysium and muscle membrane of ICTEV patients. This observation revealed that the potentially chondrogenic cells residing in the fascia of the abductor hallucis muscle may have been induced to differentiate into chondrocytes by a specific stimulation factor and therefore express high levels of the COL9A1 gene. Excessive production of COL9A1 may lead to retraction fibrosis of the abductor hallucis muscle and the supporting connective tissues. This may represent a possible causative factor for varus foot in ICTEV patients. Fibrosis may be generated by chronic damage, including muscular dystrophies. We hypothesized that the high expression of the COL9Al gene in the connective tissue of the abductor hallucis muscle results from regulation of cartilage development by important transcription factors. Specific studies have reported that the SOX9 transcription factor binds to consensus sequence pairs in the COL2A1, COL9A1 and COL27A1 genes and regulates their expression. Recently, Gu et al reported that the earliest steps of chondrogenesis may be determined by the balance between Twist1 and SOX9 expression (26).

In the current study, SOX9 and COL9A1 protein was identified to be colocalized to the connective tissue of the sarcolemma, endomysium and muscle membrane of ICTEV patients. These results indicate a correlation between $S O X 9$ and high expression of COL9A1 in the muscle samples of ICTEV patients. In addition, the promoter and coding regions of SOX 9 were analyzed for mutations in blood samples of 84 ICTEV 
patients using DGGE; however, no mutation was found. Such mutations may be revealed in larger sample sizes. Present results also revealed that the mRNA and protein expression levels of SOX9 in the abductor hallucis muscle samples of ICTEV patients were overexpressed 2-fold compared with those of the controls. mRNA levels in the ICTEV patients were correlated with those of the protein. We hypothesized that increased binding of the SOX 9 transcription factor to the upstream sequence in $C O L 9 A 1$ upregulates $C O L 9 A 1$ levels in the abductor hallucis of ICTEV patients. The significance of this potentially important mechanism remains to be determined. Although the present study used a small sample of ICTEV patients, it is reasonable to conclude that high levels of SOX9 may play a role in the development of ICTEV.

Previously, soft tissue release surgery was considered to be the best treatment method for ICTEV. However, although extensive soft tissue release provides definitive correction, the surgical procedure is associated with a number of poor, long-term complications, including muscle weakness, stiffness of ankle and subtalar joints, arthritis, pain and residual deformity (27). Thus, surgical soft tissue release has been gradually replaced by the Ponseti method of clubfoot manipulation and casting, Achilles tendon tenotomy and foot abduction bracing, which are associated with excellent short-term as well as long-term results (28). With alterations in surgical approach, soft tissue samples of ICTEV patients have become increasingly rare. In view of the relatively small number of samples in the present study, the results require confirmation in studies with larger sample sizes.

\section{Acknowledgements}

The present study was supported by a grant from the National Key Research Project of China (no. 30973140).

\section{References}

1. Dietz F: The genetics of idiopathic clubfoot. Clin Orthop Relat Res 401: 39-48, 2002.

2. Cardy AH, Barker S, Chesney D, Sharp L, Maffulli N and Miedzybrodzka Z: Pedigree analysis and epidemiological features of idiopathic congenital talipes equinovarus in the United Kingdom: a case-control study. BMC Musculoskelet Disord 8: 62, 2007.

3. Rebbeck TR, Dietz FR, Murray JC and Buetow KH: A single-gene explanation for the probability of having idiopathic talipes equinovarus. Am J Hum Genet 53: 1051-1063, 1993.

4. Wynne-Davies R: Genetic and environmental factors in the etiology of talipes equinovarus. Clin Orthop Relat Res 84: 9-13, 1972.

5. Chapman C, Stott NS, Port RV and Nicol RO: Genetics of clubfoot in Maori and Pacific people. J Med Genet 37: 680-683, 2000.

6. Miedzybrodzka Z: Congenital talipes equinovarus (clubfoot): a disorder of the foot but not the hand. J Anat 202: 37-42, 2003.

7. Liu LY, Jin CL, Cao DH, Zhao N, Lin CK and Sun KL: Analysis of association between COL9A1 gene and idiopathic congenita talipes equinovarus. Yi Chuan 29: 427-432, 2007 (In Chinese).

8. Wang LL, Fu WN, Li-Ling J,Li ZG, Li LY and Sun KL: HOXD13 may play a role in idiopathic congenital clubfoot by regulating the expression of FHL1. Cytogenet Genome Res 121: 189-195, 2008.
9. Cao D, Jin C, Ren M, Lin C, Zhang X and Zhao N: The expression of Gli3, regulated by HOXD13, may play a role in idiopathic congenital talipes equinovarus. BMC Musculoskelet Disord 10: $142,2009$.

10. Zhao Q, Eberspaecher H, Lefebvre V and De Crombrugghe B: Parallel expression of Sox 9 and Col2a1 in cells undergoing chondrogenesis. Dev Dyn 209: 377-386, 1997.

11. Sock E, Pagon RA, Keymolen K, Lissens W, Wegner M and Scherer G: Loss of DNA-dependent dimerization of the transcription factor SOX9 as a cause for campomelic dysplasia. Hum Mol Genet 12: 1439-1447, 2003.

12. Bernard P, Tang P, Liu S, Dewing P, Harley VR and Vilain E: Dimerization of SOX9 is required for chondrogenesis, but not for sex determination. Hum Mol Genet 12: 1755-1765, 2003.

13. Ng LJ, Wheatley S, Muscat GE , Conway-Campbell J, Bowles J, Wright E, Bell DM, Tam PP, Cheah KS and Koopman P: SOX9 binds DNA, activates transcription and coexpresses with type II collagen during chondrogenesis in the mouse. Dev Biol 183: 108-121, 1997.

14. Zhang P, Jimenez SA and Stokes DG: Regulation of human COL9A1 gene expression. Activation of the proximal region by SOX9. J Biol Chem 278: 117-123, 2003.

15. Rentsendorj O, Nagy A, Sinkó I, Daraba A, Barta E and Kiss I: Highly conserved proximal promoter element harbouring paired Sox9-binding sites contributes to the tissue- and developmental stage-specific activity of the matrilin-1 gene. Biochem J 389: 705-716, 2005.

16. Jenkins E, Moss JB, Pace JM and Bridgewater LC: The new collagen gene COL27A1 contains SOX9-responsive enhancer elements. Matrix Biol 24: 177-184, 2005.

17. Ross AJ, Tilman C, Yao H, MacLaughlin D and Capel B: AMH induces mesonephric cell migration in XX gonads. Mol Cell Endocrinol 211: 1-7, 2003.

18. Jakubiczka S, Schröder C, Ullmann R, et al: Translocation and deletion around SOX9 in a patient with acampomelic campomelic dysplasia and sex reversal. Sex Dev 4: 143-149, 2010.

19. van der Hout AH, van den Ouweland AM, van der Luijt RB, et al: A DGGE system for comprehensive mutation screening of BRCA1 and BRCA2: application in a Dutch cancer clinic setting. Hum Mutat 27: 654-666, 2006.

20. Kalb VF Jr and Bernlohr RW: A new spectrophotometric assay for protein in cell extracts. Anal Biochem 82: 362-371, 1977.

21. Ippolito E and Ponseti IV: Congenital club foot in the human fetus. A histological study. J Bone Joint Surg Am 62: 8-22, 1980 .

22. Ippolito E: Update on pathologic anatomy of clubfoot. J Pediatr Orthop B 4: 17-24, 1995.

23. Ionasescu V, Maynard JA, Ponseti IV and Zellweger $\mathrm{H}$ : The role of collagen in the pathogenesis of idiopathic clubfoot. Biochemical and electron microscopic correlations. Helv Paediatr Acta 29: 305-314, 1974.

24. Jackson GC, Marcus-Soekarman D, Stolte-Dijkstra I, Verrips A, Taylor JA and Briggs MD: Type IX collagen gene mutations can result in multiple epiphyseal dysplasia that is associated with osteochondritis dissecans and a mild myopathy. Am J Med GenetA 152: 863-869, 2010.

25. Li G, Zheng B, Meszaros LB, Vella JB, Usas A, Matsumoto T and Huard $\mathrm{J}$ : Identification and characterization of chondrogenic progenitor cells in the fascia of postnatal skeletal muscle. J Mol Cell Biol 3: 369-377, 2011.

26. Gu S, Boyer TG and Naski MC: Basic helix-loop-helix transcription factor twist 1 inhibits the transactivation function of the master chondrogenic regulator Sox9. J Biol Chem 287: 21082-21092, 2012.

27. Ippolito E, Farsetti P, Caterini R and Tudisco C: Long-term comparative results in patients with congenital clubfoot treated with two different protocols. J Bone Joint Surg Am 85: 1286-1294, 2003.

28. Ponseti IV: Treatment of congenital club foot. J Bone Joint Surg Am 74: 448-454, 1992. 